\title{
Classical SU(3) Gauge Field as a Dark Matter
}

\author{
Vladimir Dzhunushaliev ${ }^{1,2}$ \\ ${ }^{1}$ Institute for Basic Research, Eurasian National University, Astana, Kazakhstan \\ ${ }^{2}$ Department of Theoretical and Nuclear Physics, Al-Farabi Kazakh National University, Almaty, Kazakhstan \\ Email: v.dzhunushaliev@gmail.com
}

Received June 5, 2013; revised July 18, 2013; accepted August 4, 2013

Copyright (C) 2013 Vladimir Dzhunushaliev. This is an open access article distributed under the Creative Commons Attribution License, which permits unrestricted use, distribution, and reproduction in any medium, provided the original work is properly cited.

\begin{abstract}
The model of dark matter is presented where the dark matter is a classical gauge field. A spherical symmetric solution of Yang-Mills equation is obtained. The asymptotic behavior of the gauge fields and matter density is investigated. It is shown that the distribution of the matter density allows us interpret it as the dark matter. The fitting of a typical rotational curve with the rotational curve created by the spherical solution of SU(3) Yang-Mills equation is made
\end{abstract}

Keywords: Dark Matter; Color Gauge Field

\section{Introduction}

One can give such definition for a dark matter [1]: “... dark matter is matter of unknown composition that does not emit or reflect enough electromagnetic radiation to be observed directly, but whose presence can be inferred from gravitational effects on visible matter." The nature of the dark matter of the Universe is one of the most challenging problems facing modern physics. Following to $\mathrm{L}$. Smolin [2] there exist five great problems in the modern theoretical physics:

Problem 1. Combine general relativity and quantum theory into a single theory that can claim to be the complete theory of nature. This is called the problem of quantum gravity.

Problem 2. Resolve the problems in the foundations of quantum mechanics, either by making sense of the theory as it stands or by inventing a new theory that does make sense.

Problem 3. Determine whether or not the various particles and forces can be unified in a theory that explains them all as manifestations of a single, fundamental entity.

Problem 4. Explain how the values of the free constants in the standard model of particle physics are chosen in nature.

Problem 5. Explain dark matter and dark energy. Or, if they don't exist, determine how and why gravity is modified on large scales. More generally, explain why the constants of the standard model of cosmology, including the dark energy, have the values they do.
The problem of the dark matter is the fifth one. Direct observational evidence for dark matter is found from a variety of sources:

- On the scale of galactic halos, the observed flatness of the rotation curves of spiral galaxies is a clear indicator for dark matter.

- The measured orbital velocities of galaxies within galactic clusters have been found to be consistent with dark matter observations.

- In clusters of galaxies there is a hot intracluster gas. Its temperature allows to measure gravitational potential of a cluster. These data are in agreement with measurements of galaxies speeds and show presence of dark matter.

- The direct evidence of dark matter has been obtained through the study of gravitational lenses.

One of the strongest pieces of evidence for the existence of dark matter is following. Let us consider a rotational velocity $v(r)$ of stars in a galaxy. According to Newton law

$$
v^{2}(r) \propto G_{N} \frac{M(r)}{r}
$$

where $M(r)$ is the mass at a given distance $r$ from the center of a galaxy; $G_{N}$ is the Newton gravitational constant. The rotational velocity, is measured $[3,4]$ by observing $21 \mathrm{~cm}$ emission lines in HI regions (neutral hydrogen) beyond the point where most of the light in the galaxy ceases. Schematically a typical rotation curves of spiral galaxies is shown in Figure 1 (for details, see Refs. [5,6]). 


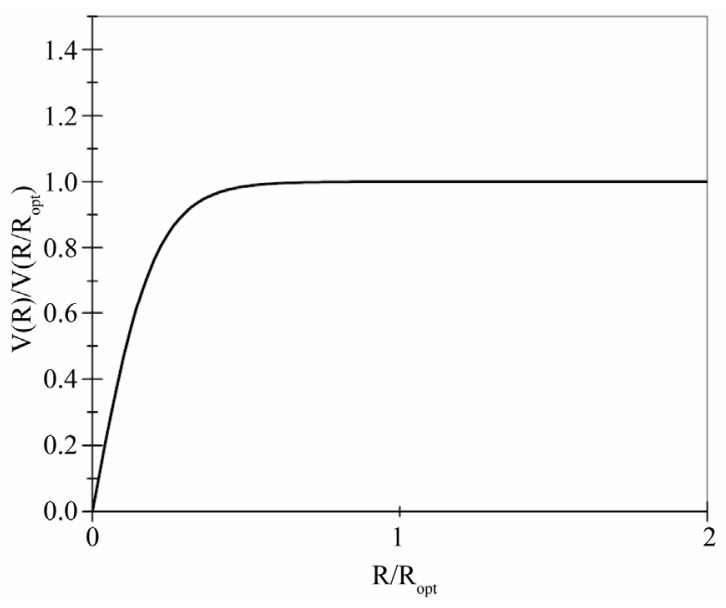

Figure 1. Schematical rotation curve of spiral galaxies.

If the bulk of the mass is associated with light, then beyond the point where most of the light stops, $M(r)$ would be a constant and $v(r)^{2} \propto 1 / r$. This is not the case, as the rotation curves appear to be flat, i.e., $v(r)$ : constant outside the core of the galaxy. This implies that $M(r) \propto r$ beyond the point where the light stops. This fact is the evidence of the existence of dark matter. The detailed review for the experimental evidence of dark matter can be found in [7].

Several categories of dark matter have been postulated: (a) baryonic dark matter; (b) non-baryonic dark matter. The non-baryonic dark matter can be divided into three different types: (b1) hot dark matter-nonbaryonic particles that move ultrarelativistically[8]; (b2) warm dark matter-nonbaryonic particles that move relativistically; (b3) cold dark matter-nonbaryonic particles that move non-relativistically[9].

Another approach for the resolution of the dark matter problem is based on a modification of Newton's law or of general relativity, have been proposed to explain the behavior of the galactic rotation curves: (a) a modified gravitational potential [10,11]; (b) the Poisson equation for the gravitational potential is replaced by another equation [12-17]; (c) alternative theoretical models to explain the galactic rotation curves have been elaborated by Mannheim [17,18], Moffat and Sokolov [19] and Roberts [20]; (d) The idea that dark matter is a result of the bulk effects in brane world cosmological models was considered in [21,22].

All above mentioned approaches to the resolution dark matter problem are based on the assumption that the dark matter is one or another kind of quantum particles. The problem for this approach is that the most of these particles are hypothetical ones: till now these particles were not observed in the nature and in spite of general enthusiasm we do not have any confidence that these particles will be discovered.
Here we propose the idea that the dark matter is a classical gauge field. This approach is based on the fact that in the consequence of the nonlinearity of Yang-Mills equations there exist a spherically symmetric solution without sources (color charge). The matter density in such solution is $\propto r^{-2 \alpha}$ with $\alpha<2$ that radically differs from the distribution matter density for Coulomb solution. Thus the proposed idea is that some galaxies are immersed in a cloud of a classical gauge field. The SU(3) classical gauge field does not interact with ordinary matter because ordinary matter is colorless. Thus one can suppose that SU(3) gauge field can be invisible matter in galaxies. The problem for such consideration is why the gauge field does not fill all Universe? The probable answer is that, in certain circumstances, the gauge field goes from a classical phase into a quantum phase. Probably such transition takes place at some distance from the center of the galaxy.

Let us note that in Refs. [23-27] the similar approach for a dark energy is considered. In Ref. [23] it is shown that the Born-Infeld quantum condensate can play the role of dark energy in the present-day universe. In Ref. [24], it is demonstrated that both inflation and the latetime acceleration of the universe can be realized in modified Maxwell- $F(R)$ and Yang-Mills- $F(R)$ gravities. In Refs. [25-27], it is supposed that the dark energy is a condensate of Yang-Mills gauge field where the effective Lagrangian density of the YM field is calculated up to 1-loop order [28,29]. With the logarithmic dependence on the field strength, the effective Lagrangian has a form similar to the Coleman-Weinberg scalar effective potential [30].

\section{Classical Gauge Theory}

In this section we would like to give a short introduction to $\mathrm{SU}(N)$ Yang-Mills gauge theory. The corresponding Lagrangian is

$$
\mathrm{L}=F_{\mu \nu}^{A} F^{A \mu \nu}
$$

where $A, B, C=1,2, \cdots, N$ are the $\mathrm{SU}(N)$ color indexes; $F_{\mu v}^{B}=\partial_{\mu} A_{v}^{B}-\partial_{v} A_{\mu}^{B}+g f^{\mathrm{BCD}} A_{\mu}^{C} A_{v}^{D}$ is the field strength; $A_{\mu}^{B}$ is the $\mathrm{SU}(N)$ gauge potential; $g$ is the coupling constant; $f^{B C D}$ are the structure constants for the $\mathrm{SU}(N)$ gauge group. The corresponding Yang-Mills field equations are

$$
\partial_{v} F^{A \mu \nu}=0 .
$$

Here we will consider $N=3$ case of the Yang-Mills equations. Equations (3) are nonlinear generalizations of Maxwell equations. The spherally symmetric static solution in electrodynamics is Coulomb potential. Well known spherically symmetric static solution for the $\mathrm{SU}(2)$ Yang-Mills equations are famous monopole and instanton solutions. The monopole solution has finite 
energy and is a special solution for corresponding equations. For our goals we will consider practically the same equations as for monopole but with different boundary conditions. Strictly speaking the solutions of Equations (4)-(11) for almost all boundary conditions are singular (the energy is infinite) and only for some special choice of boundary conditions we have regular solution (monopole solution with finite energy). In this work we use solution with infinite energy but we assume that at some distance from the origin the classical phase undergoes a transition to a quantum phase.

\subsection{Ansatz for the Gauge Potential}

The classical SU(3) Yang-Mills gauge field $A_{\mu}^{B}$ we choose in the following form [31]

$$
\begin{gathered}
A_{0}^{2}=-2 \frac{z}{g r^{2}} \chi(r), \\
A_{0}^{5}=2 \frac{y}{g r^{2}} \chi(r), \\
A_{0}^{7}=-2 \frac{x}{g r^{2}} \chi(r), \\
A_{i}^{2}=2 \frac{\varepsilon_{3 i j} x^{j}}{g r^{2}}[h(r)+1], \\
A_{i}^{5}=-2 \frac{\varepsilon_{2 i j} x^{j}}{g r^{2}}[h(r)+1], \\
A_{i}^{7}=2 \frac{\varepsilon_{1 i j} x^{j}}{g r^{2}}[h(r)+1]
\end{gathered}
$$

here $A_{\mu}^{2,5,7} \in \mathrm{SU}(2) \subset \mathrm{SU}(3)$; the $\mathrm{SU}(2)$ is the subgroup of $\mathrm{SU}(3) ; \mu=0,1,2,3$ is the space-time index and $i, j, k=1,2,3$ are space indexes. The remaining components are belong to the coset $\mathrm{SU}(3) / \mathrm{SU}(2)$

$$
\begin{aligned}
& \left(A_{0}\right)_{\alpha, \beta}=2\left(\frac{x^{\alpha} x^{\beta}}{r^{2}}-\frac{1}{3} \delta^{\alpha \beta}\right) \frac{w(r)}{g r}, \\
& \left(A_{i}\right)_{\alpha \beta}=2\left(\varepsilon_{i s \alpha} x^{\beta}+\varepsilon_{i s \beta} x^{\alpha}\right) \frac{x^{s}}{g r^{3}} v(r),
\end{aligned}
$$

here $\varepsilon_{i j k}$ is the absolutely antisymmetric Levi-Civita tensor. The ansatz (4)-(11) is the SU(3) generalization of ansatz for the SU(2) monopole [32,33]. The SU(3) ansatz gives us a spherically symmetric energy distribution that is necessary to describe a spherical dark matter distribution in a galaxy.

The coset components $\left(A_{\mu}\right)_{\alpha \beta}$ are written in the matrix form and by definition

$$
\left(A_{\mu}\right)_{\alpha \beta}=\sum_{m=1,3,4,6,8} A_{\mu}^{m}\left(T^{m}\right)_{\alpha, \beta}
$$

where $T^{B}=\frac{\lambda^{B}}{2}$ are the $\mathrm{SU}(3)$ generators, $\lambda^{B}$ are the Gell-Mann matrices.

\subsection{Yang-Mills Equations}

The corresponding Yang-Mills Equations (3) with the potential (4)-(11) and $(\chi(r)=h(r)=0)$ are

$$
\begin{gathered}
x^{2} w^{\prime \prime}=6 w v^{2}, \\
x^{2} v^{\prime \prime}=v^{3}-v-v w^{2}
\end{gathered}
$$

here we introduce the dimensionless radius $x=r / r_{0}, r_{0}$ is an arbitrary constant. The asymptotic behavior of the functions $v(x), w(x)$ by $x \gg 1$ are [34]

$$
\begin{aligned}
& v(x) \approx A \sin \left(x^{\alpha}+\phi_{0}\right), \\
& w(x) \approx \pm\left[\alpha x^{\alpha}+\frac{\alpha-1}{4} \frac{\cos \left(2 x^{\alpha}+2 \phi_{0}\right)}{x^{\alpha}}\right] \\
& 3 A^{2}=\alpha(\alpha-1)
\end{aligned}
$$

with $\alpha>1$. The energy density $\varepsilon(x)$ is

$$
\begin{aligned}
& \varepsilon(r)=-F_{0 i}^{a} F^{a 0 i}+\frac{1}{4} F_{i j}^{a} F^{a i j} \\
& =\frac{1}{g^{2} r_{0}^{4}}\left[4 \frac{v^{\prime 2}}{x^{2}}+\frac{2}{3} \frac{\left(x w^{\prime}-w\right)^{2}}{x^{4}}+2 \frac{\left(v^{2}-1\right)^{2}}{x^{4}}+4 \frac{v^{2} w^{2}}{x^{4}}\right] \\
& =\frac{1}{g^{2} r_{0}^{4}} \varepsilon(x) .
\end{aligned}
$$

Using the asymptotic behavior of the gauge potential (15) (16) the asymptotic behavior of the energy density is.

$$
\varepsilon_{\infty}(x) \approx \frac{2}{3} \alpha^{2}(\alpha-1)(3 \alpha-1)\left(\frac{r}{r_{0}}\right)^{2 \alpha-4} .
$$

\subsection{Numerical Investigation}

In this subsection we present the typical numerical solution of Equation's (13) and (14). In the consequence of the occurrence of the factor $x^{2}$ in the front of left hide side of Equations (13) and (14) we have to start the numerical calculations not from $x=0$ but from from $x=\delta=1$. To do that we search an approximate solution near to the origin in te form

$$
\begin{aligned}
& v(r) \\
= & 1+\frac{1}{2}\left(r_{0}^{2} v_{2}\right)\left(\frac{r}{r_{0}}\right)^{2}+\frac{1}{4 !}\left(r_{0}^{4} v_{4}\right)\left(\frac{r}{r_{0}}\right)^{4}+\mathrm{O}\left[\left(\frac{r}{r_{0}}\right)^{6}\right] \\
= & 1+v_{2}^{\prime} \frac{x^{2}}{2}+v_{4}^{\prime} \frac{x^{4}}{4 !}+\mathrm{O}\left(x^{4}\right)
\end{aligned}
$$




$$
\begin{aligned}
w(r) & =\frac{1}{6}\left(r_{0}^{3} w_{3}\right)\left(\frac{r}{r_{0}}\right)^{3} \\
& +\frac{1}{5 !}\left(r_{0}^{5} w_{5}\right)\left(\frac{r}{r_{0}}\right)^{5}+\mathrm{O}\left[\left(\frac{r}{r_{0}}\right)^{7}\right] \\
& =w_{3}^{\prime} \frac{x^{3}}{6}+w_{5}^{\prime} \frac{x^{5}}{5 !}+\mathrm{O}\left(x^{7}\right) .
\end{aligned}
$$

After the substitution into Yang-Mills Equations (13) and (14), we have

$$
\begin{aligned}
v_{4} & =\frac{9}{5} v_{2}^{2}, \\
w_{5} & =\frac{60}{7} v_{2} w_{3}
\end{aligned}
$$

and so on for the next $v_{i}, w_{j}, i>4, j>7$. It means that $v_{2}, w_{3}$ are free parameters defining the solution. YangMills equations are conformally invariant under $\boldsymbol{r} \rightarrow r_{0} \boldsymbol{r}$, i.e. $r_{0}$ is a free parameter also. We use following choice of the parameter $r_{0}$

$$
\text { either } r_{0}^{2}=\frac{1}{v_{2}} \text { or } r_{0}^{3}=\frac{1}{w_{3}} \text {. }
$$

For the numerical calculations we choose the parameter $r_{0}$ as

$$
r_{0}=\frac{1}{w_{3}^{1 / 3}} .
$$

The typical behavior of functions $v(x)$ and $w(x)$ is presented in Figure 2, that is in agreement with the asymptotic behavior (15) and (16).

The mass density $\rho(r)$ is

$$
\rho(r)=\frac{1}{g^{2} c^{2} r_{0}^{4}} \rho(x)
$$

where $\rho(x)=\varepsilon(x)$ and $\varepsilon(x)$ is given in Equation (18). The profile of the dimensionless energy density $\varepsilon(x)$ in Figure 3 is presented.

\subsection{The Comparison with a Universal Rotation Curve of Spiral Galaxies}

The idea presented in this work is that in a galaxy there exists an ordinary visible matter (barionic matter which glows) and an invisible matter (classic gauge field which does not interact with electromagnetic waves). The visible matter is immersed into a cloud of the classical gauge field. The main goal of this work is to compare the experimental rotational curve for stars in the galaxy outside light core with the rotational curve created by the distribution of the classical gauge field.

In Ref. [35] a Universal Rotation Curve of spiral galaxies is offered that describes any rotation curve at any radius with a very small cosmic variance

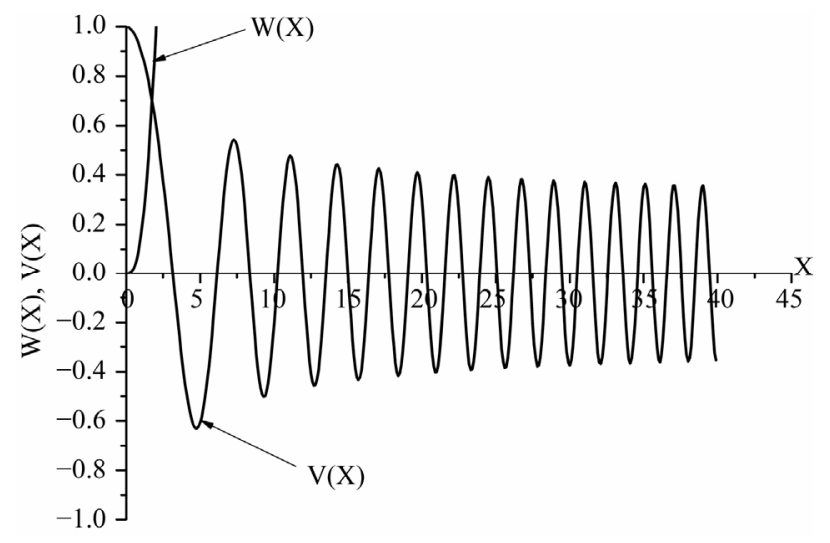

Figure 2. The profiles of functions $w(x), v(x), v_{2}^{\prime}=-0.2$, $w_{3}^{\prime}=1$.

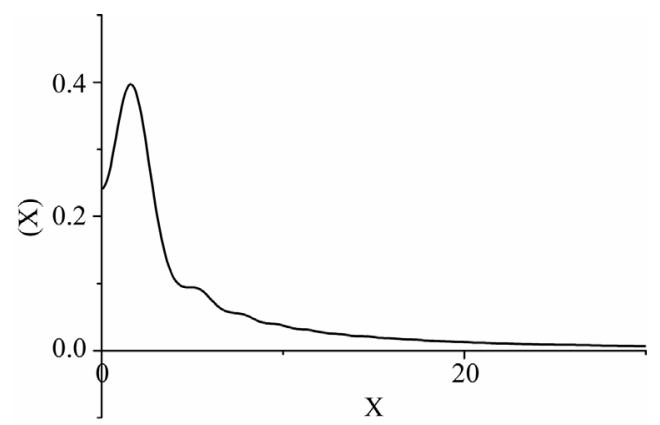

Figure 3. The profile of the dimensionless energy density $\varepsilon(x)$.

$$
\begin{aligned}
& {\left[\frac{V_{U R C}^{2}\left(\frac{r}{R_{o p t}}\right)}{V\left(R_{o p t}\right)}\right]^{2}} \\
& =\left(0.72+0.44 \log \frac{L}{L_{*}}\right) \frac{1.97 X^{1.22}}{\left(X^{2}+0.78^{2}\right)^{1.43}} . \\
& +1.6 \mathrm{e}^{-0.4\left(L / L_{*}\right)} \frac{X^{2}}{X^{2}+1.5^{2}\left(\frac{L}{L_{*}}\right)^{0.4}} \\
& =V_{L M}^{2}+V_{D M}^{2}, \operatorname{Km} \\
& {\left[\begin{array}{l}
V_{L M}^{2}\left(\frac{r}{R_{o p t}}\right) \\
V\left(R_{o p t}\right)
\end{array}\right]^{2}} \\
& =\left(0.72+0.44 \log \frac{L}{L_{*}}\right) \frac{1.97 X^{1.22}}{\left(X^{2}+0.78^{2}\right)^{1.43}},
\end{aligned}
$$




$$
\left[\frac{V_{D M}^{2}\left(\frac{r}{R_{o p t}}\right)}{V\left(R_{o p t}\right)}\right]^{2}=1.6 \mathrm{e}^{-0.4\left(L / L_{*}\right)} \frac{X^{2}}{X^{2}+1.5^{2}\left(\frac{L}{L_{*}}\right)^{0.4}}
$$

where $R_{\text {opt }} \equiv 3.2 R_{D}$ is the optical radius and $R_{D}$ is the disc exponential length-scale; $X=r / R_{\text {opt }} ; L$ is the luminosity; the first term $V_{L M}^{2}$ is the rotation curve for the light matter and the second term $V_{D M}^{2}$ the rotation curve for the dark matter. Our goal is to compare the rotation curve for the color fields

$$
\begin{aligned}
V^{2}(r) & =G_{N} \frac{\mathrm{M}(r)}{r}=4 \pi G_{N} \frac{1}{r} \int_{0}^{r} r^{2} \rho(r) \mathrm{d} r \\
& =\frac{G_{N} \hbar}{c^{3}} \frac{c^{2}}{g^{\prime 2} r_{0}^{2}} \frac{\mathrm{M}(x)}{x}=\left[\frac{1}{g^{\prime 2}}\left(\frac{l_{P l}}{r_{0}}\right)^{2} \frac{\mathrm{M}(x)}{x}\right] c^{2}
\end{aligned}
$$

where $\mathrm{M}(x)$ is the dimensionless mass of the color fields $A_{\mu}^{B}$ inside the sphere of radius $r=x r_{0}$;

$g^{\prime 2}=g^{2} c \hbar / 4 \pi$ is the dimensionless coupling constant with the experimental rotation curve for the dark matter (29).

Far away from the center the dimensionless energy density $\varepsilon_{\infty}(x)$ is presented in Equation (19). In order to fit Equation (19) we break up Equation (30) into two terms

$$
\begin{aligned}
& V^{2} \\
& =c^{2} \frac{1}{g^{\prime 2}}\left(\frac{l_{P l}}{r_{0}^{2}}\right)^{2} \frac{1}{x}\left(\int_{0}^{x} x^{2}\left[\varepsilon(x)-\varepsilon_{\infty}(x)\right] \mathrm{d} x+\int_{x_{1}}^{x} x^{2} \varepsilon_{\infty}(x) \mathrm{d} x\right) \\
& \approx\left[c^{2} \frac{1}{g^{\prime 2}}\left(\frac{l_{P l}}{r_{0}^{2}}\right)^{2} \frac{1}{x} \int_{0}^{x} x^{2} \varepsilon_{\infty}(x) \mathrm{d} x\right]-V_{0}^{2}
\end{aligned}
$$

where

$$
V_{0}^{2}=c^{2} \frac{1}{g^{\prime 2}}\left(\frac{l_{P l}}{r_{0}^{2}}\right)^{2} \frac{1}{x} \int_{0}^{x} x^{2}\left[\varepsilon_{\infty}(x)-\varepsilon(x)\right] \mathrm{d} x .
$$

The numerical value of $V_{0}^{2}$ is defined near to the center of galaxy where according to Equation (32) the difference $\varepsilon_{\infty}(x)-\varepsilon(x)$ is maximal. Thus the asymptotic behavior of the rotation curve for the domain filled with the $\mathrm{SU}(3)$ gauge field is

$$
V^{2} \approx\left[\frac{2}{3} \frac{1}{g^{\prime 2}}\left(\frac{l_{P I}}{r_{0}}\right)^{2} \frac{\alpha^{2}(\alpha-1)(3 \alpha-1)}{2 \alpha-1}\left(\frac{r}{r_{0}}\right)^{2 \alpha-2}\right] c^{2}-V_{0}^{2} .
$$

In Figure 4 the profiles of the dark matter rotational curves (29) (for different values $L / L_{*}=0.5,1.0,15$ ) and fitting curves (33) are presented. The value of parameter

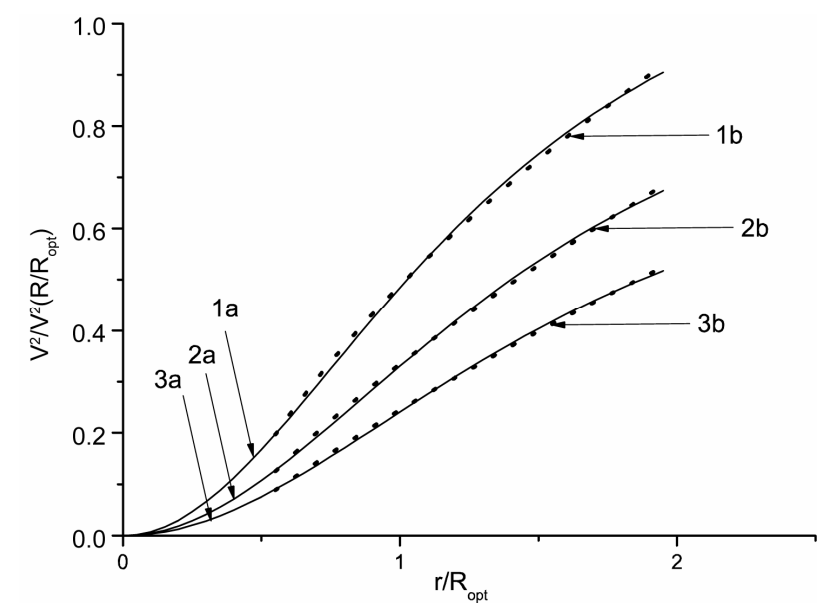

Figure 4. The comparison of DM rotation curve (29) for different values $L / L_{*}=0.5,1.0,1.5$; curves 1a, 2 a, 3a correspondingly) with the rotation curve (33) (curves $1 \mathrm{~b}, 2 \mathrm{~b}, 3 \mathrm{~b}$ correspondingly) for the $\mathrm{SU}(3)$ classical color field (4)-(9). $\alpha \approx$ $1.21,1.31,1.38, g^{\prime}=1, R_{\text {opt }}=20 \mathrm{KPs}=6 \times 10^{17} \mathrm{~m}, V_{\text {opt }}=100$ $\mathrm{Km} / \mathrm{s}$.

$\alpha$ is given from Section 2.3. The details of fitting

$$
\begin{aligned}
& r_{0} \approx 1.18 \times 10^{-21} \mathrm{~cm}, V_{0} \approx 78.6 \mathrm{Km} / \mathrm{s}, \\
& \alpha=1.21 ; \frac{L}{L_{*}}=0.5, \\
& r_{0} \approx 2.01 \times 10^{-18} \mathrm{~cm}, V_{0} \approx 32.25 \mathrm{Km} / \mathrm{s}, \\
& \alpha=1.31 ; \frac{L}{L_{*}}=1.0, \\
& r_{0} \approx 8.04 \cdot 10^{-17} \mathrm{~cm}, V_{0} \approx 18.5 \mathrm{Km} / \mathrm{s}, \\
& \alpha=1.38 ; \frac{L}{L_{*}}=1.5
\end{aligned}
$$

in Appendix (B) are presented. The physical meaning of $r_{0}$ is that by $r>r_{0}$ one can use the asymptotical behavior (15) and (16). Small values of $r_{0}$ (34)-(36) mean that we can use the asymptotical behavior (15) and (16) practically in the whole region of radius $r$.

\section{Cut-Off the Region Filled with Classical Gauge Field}

The gauge field distribution considered here has an infinite energy in the consequence of the asymptotic behavior (18) of the energy density (18). Consequently it is necessary to have a mechanism to cut-off the distribution of the classical gauge fields in the space. In our opinion it can happen in the following way. At some distance from the origin the gauge field undergoes the transition from the classical state to quantum one and the quantum field tends very fast to its vacuum expectation value. It is very important to note that the gauge field in the vacuum state must be described in a nonperturbative 
manner.

The physical reason why such transition takes place is following. As we see from Equations (15) and (16) and Figure $\mathbf{2}$ asymptoticly the gauge potentials are oscillating functions with increasing frequency. Far away from the origin the frequency is so big that it is necessary quantum fluctuations take into account. In this way the transition from the classical state to quantum one takes place.

To estimate a transition radius we follow to the Heisenberg uncertainty principle

$$
\frac{1}{C} \Delta F_{t i}^{a} \Delta A^{a i} \Delta V \approx \hbar
$$

here $\Delta F_{t i}^{a}$ is a quantum fluctuation of the color electric field $F_{t i}^{a} ; \Delta A^{a i}$ is a quantum fluctuation of the color electric potential $A^{a i} ; \Delta V$ is the volume where the quantum fluctuations $\Delta F_{t i}^{a}$ and $\Delta A^{a i}$ takes place and not any summation over repeating indexes.

For the ansatz (10) and (11)

$$
F_{t \theta}^{2}=-\frac{2 \sin \theta}{g} \frac{v w}{r} .
$$

Let us to introduce the physical component of the $F_{t \theta}^{2}$

$$
\left|\tilde{F}_{t \theta}^{2}\right|=\sqrt{F_{t \theta}^{2} F_{t}^{2 \theta}}=\frac{2 \sin \theta}{g} \frac{v w}{r^{2}} .
$$

To an accuracy of a numerical factor the fluctuations of the SU(3) color electric field are

$$
\Delta \tilde{F}_{t \theta}^{2} \approx \frac{1}{g} \frac{1}{r^{2}}(\Delta v w+v \Delta w) .
$$

For the ansatz (10) and (11)

$$
\begin{gathered}
A_{\theta}^{2}=0, \\
A_{\theta}^{1,3,4,6,8} \approx \frac{1}{g} v .
\end{gathered}
$$

The physical components of the gauge potential $A_{\theta}^{1,3,4,6,8}$

$$
\left|\tilde{A}_{\theta}^{1,3,4,6,8}\right|=\sqrt{A_{\theta}^{1,3,4,6,8} A^{1,3,4,6,8 ; \theta}} \approx \frac{1}{g} \frac{v}{r} .
$$

Now we assume that the quantum fluctuations $\Delta \tilde{A}_{\theta}^{2}$ of the component $\tilde{A}_{\theta}^{2}$ have the same order as the quantum fluctuations of the components $\tilde{A}_{\theta}^{1}$

$$
\Delta \tilde{A}_{\theta}^{2} \approx \Delta \tilde{A}_{\theta}^{1} \approx \frac{1}{g} \frac{\Delta v}{r} .
$$

The volume $\Delta V$ where supposed quantum fluctuations take place is

$$
\Delta V=4 \pi r^{2} \Delta r .
$$

The period of space oscillations by $r \gg r_{0}$ can be defined in the following way

$$
(x+\lambda)^{\alpha}-x^{\alpha} \approx \alpha \frac{\lambda}{x^{1-\alpha}}=2 \pi ; \quad x=\frac{r}{r_{0}} .
$$

We suppose that the place where the SU(3) classical color field becomes quantum one is defined as the place where the quantum fluctuations in the volume $\Delta V=4 \pi r^{2} \Delta r$ with

$$
\frac{\Delta r}{r_{0}} \approx \lambda \approx \frac{1}{\alpha} \frac{2 \pi}{x^{\alpha-1}}
$$

of the corresponding field becomes comparable with magnitude of these fields

$$
\Delta v \approx v, \quad \Delta w \approx w
$$

Substituting of Equations (40), (15)-(16), (44), (45), (47) and (48) into Equation (37) we obtain

$$
\left(\frac{g^{\prime}}{A}\right)^{2} \approx 2 \pi
$$

where $\frac{1}{g^{\prime}}=\frac{4 \pi}{g^{2} \hbar c}$ is the dimensionless coupling constant that is similar to the fine structure constant in quantum electrodynamics $\alpha=\frac{e^{2}}{\hbar c}$. In quantum chromodynamics $\beta=1 / g^{\prime 2} \geq 1$. If we choose $1 / g^{\prime} \approx 1$ and from Figure 2 we take $A \approx 0.4$ we see that

$$
\left(\frac{g^{\prime}}{A}\right)^{2} \approx 6.25
$$

that is comparable with $2 \pi \approx 6.28$.

Thus in this section we have shown that if the condition (49) is true then at some distance from the center the transition from the classical phase to quantum one occurs. Unfortunately the rough estimation presented in this section does not allow us to calculate the radius where such transition takes place. For the exact evaluation of the place where such transition happens it is necessary to have non-perturbative quantization methods which are missing at the moment.

\section{Invisibility of the Color Dark Matter}

The main question in any dark matter model is its invisibility. For the model presented here the answer is very simple: the SU(3) color matter (dark matter in this context) is invisible because color gauge fields interact with color charged particles only. But at the moment in the nature we do not know any particles with $\mathrm{SU}(3)$ color charge. In principle such particles can be SU(3) monopoles but up to now the monopoles are not experimentally registered.

For more details we write SU(3) Lagrangian interact- 
ing with matter

$$
\mathrm{L}_{Q C D}=-\frac{1}{2} \operatorname{tr} F_{\mu \nu}^{A} F^{A \mu \nu}+\sum_{k}^{n_{f}} \bar{q}\left(i \gamma^{\mu} D_{\mu}-m_{k}\right) q_{k},
$$

where

$$
\begin{aligned}
D_{\mu} q_{k} & =\left(\partial_{\mu}-i g A_{\mu}\right) q \\
A_{\mu} & =\sum_{B=1}^{8} A_{\mu}^{B} \frac{\lambda^{B}}{2}
\end{aligned}
$$

$D_{\mu}$ is the covariant derivative; $A_{m} \mu$ is gauge potential in the matrix form. From the term $i g \bar{q} A_{\mu} q$ in Equation (52) we see that the $\mathrm{SU}(3)$ color field has an interaction with quarks only. But the quarks are not observable in the nature. The baryon matter is colorless in the consequence with the confinement of quarks in hadrons and therefore the color dark matter does not interact with the light (photons). As we see above the gauge dark matter can be seen in during of its gravitational field.

Thus it is interesting that the problem of the dark matter in astrophysics is connected with the problem of confinement in high energy physics.

\section{Conclusion}

In this work we have suggested the idea that the dark matter model is $\mathrm{SU}(3)$ gauge field. We have shown that in SU(3) Yang-Mills theory there exists a spherical symmetric distribution of the gauge potential with slow decreasing matter density. The asymptotic behavior of the density allow us to describe the rotational curve for the stars in elliptic galaxies. The fitting of the typical rotational curve gives us parameters which have a good agreement with the parameters of above mentioned spherical solution of Yang-Mills equations.

\section{Acknowledgements}

I am gratefully acknowledging support provided by the grants No. 378 in fundamental research in natural sciences by the Ministry of Education and Science of Kazakhstan and the Volkswagen Foundation.

\section{REFERENCES}

[1] Wikipedia, "Dark-Matter," 2013. http://en.wikipedia.org/wiki/Dark_matter

[2] L. Smolin, “The Troubles with Physics,” A Mariner book Houghton Mifflin Company, Boston, New York, 2007.

[3] S. M. Faber and J. J. Gallagher, Annual Review of Astronomy and Astrophysics, Vol. 17, 1979, pp.135-187. doi:10.1146/annurev.aa.17.090179.001031

[4] A. Bosma, The Astrophysical Journal, Vol. 86, 1981, pp. 1825-1837.

[5] M. Persic and P. Salucci, The Astrophysical Journal Sup- plement Series, Vol. 99, 1995, pp. 501-520.

[6] M. Persic, P. Salucci and F. Stel, MNRAS, Vol. 281, 1996, pp. 27P-35P. doi:10.1093/mnras/278.1.27

[7] K. A. Olive, "Dark Matter," Lectures Given at Theoretical Advanced Study Institute in Elementary Particle Physics (TASI 2002): Particle Physics and Cosmology: The Quest for Physics Beyond the Standard Model(s), Boulder, Colorado, 2-28 June 2002.

[8] K. Bamba, S. Nojiri and S. D. Odintsov, Physical Review D, Vol. 77, 2008, pp. 123532-123540. doi:10.1103/PhysRevD.77.123532

[9] Y. Zhang, Physics Letters B, Vol. 340, 1994, pp. 18-30. doi:10.1016/0370-2693(94)91291-2

[10] R. H. Sanders, Astronomy \& Astrophysics, Vol. 136, 1984, pp. L21-L33.

[11] R. H. Sanders, Astronomy \& Astrophysics, Vol. 154, 1986, pp. 135-141.

[12] M. Milgrom, The Astrophysical Journal, Vol. 270, 1983, pp. 365-384. doi:10.1086/161130

[13] J. Bekenstein and M. Milgrom, The Astrophysical Journal, Vol. 286, 1984, pp. 7-15.

[14] M. Milgrom, New Astronomy Reviews, Vol. 46, 2002, pp. 741-752. doi:10.1016/S1387-6473(02)00243-9

[15] M. Milgrom, The Astrophysical Journal, Vol. 599, 2003, pp. L25-L31. doi:10.1086/381138

[16] J. D. Bekenstein, Physical Review, Vol. D70, 2004, pp. 083509-083521.

[17] P. D. Mannheim, The Astrophysical Journal, Vol. 419, 1993, pp. 150-160. doi:10.1086/173468

[18] P. D. Mannheim, The Astrophysical Journal, Vol. 479, 1997, pp. 659-671. doi:10.1086/303933

[19] J. W. Moffat and I. Y. Sokolov, Physics Letters, Vol. B378, 1996, pp. 59-67. doi:10.1016/0370-2693(96)00366-8

[20] M. D. Roberts, General Relativity and Gravitation, Vol. 36, 2004, pp. 2423-2430. doi:10.1023/B:GERG.0000046830.85831.4a

[21] T. Harko and M. K. Mak, Annals of Physics, Vol. 319, 2005, pp. 471-482. doi:10.1016/j.aop.2005.04.010

[22] T. Harko and K. S. Cheng, The Astrophysical Journal, Vol. 636, 2005, pp. 8-15. doi:10.1086/498141

[23] E. Elizalde, J. E. Lidsey, S. Nojiri and S. D. Odintsov, Physics Letters B, Vol. 574, 2003, pp. 1-12. doi:10.1016/j.physletb.2003.08.074

[24] K. Bamba and S. D. Odintsov, JCAP, Vol. 0804, 2008, pp. 024-030.

[25] Y. Zhang, General Relativity and Gravitation, Vol. 34, 2002, pp. 2155-2166. doi:10.1023/A:1021195720598

[26] W. Zhao and Y. Zhang, Classical and Quantum Gravity, Vol. 23, 2006, pp. 3405-3414. doi:10.1088/0264-9381/23/10/011

[27] W. Zhao and Y. Zhang, Physics Letters B, Vol. 640, 2006, pp. 69-74. doi:10.1016/j.physletb.2006.07.052

[28] H. Pagels and E. Tomboulis, Nuclear Physics B, Vol. 143, 
1978, pp. 485-490. doi:10.1016/0550-3213(78)90065-2

[29] S. Adler, Physical Review D, Vol. 23, 1981, pp. 29052916. doi:10.1103/PhysRevD.23.2905

[30] S. Coleman and E. Weinberg, Physical Review D, Vol. 7, 1973, pp. 1888-1897. doi:10.1103/PhysRevD.7.1888

[31] E. Corrigan, D. I. Olive, D. B. Farlie and J. Nuyts, Nuclear Physics, Vol. B106, 1976, pp. 475-492.

[32] G.'t Hooft, Nuclear Physics, Vol. B79, 1976, pp. 276-280. doi:10.1016/0550-3213(74)90486-6

[33] A. M. Polyakov, JETP Letters, Vol. 20, 1974, Article ID: 194201.

[34] V. D. Dzhunushaliev and D. Singleton, "Confining Solutions of SU(3) Yang-Mills Theory,” In: V. V. Dvoeglazov,
Ed., Contribution to Contemporary Fundamental Physics, Nova Science Publishers, Hauppauge, 1999, pp. 336-346.

[35] M. Persic, P. Salucci and F. Stel, "The Universal rotation Curve of Spiral Galaxies: 1. The Dark Matter Connection," Monthly Notices of the Royal Astronomical Society, Vol. 281, No. 1, 1996, pp. 27-47. doi:10.1093/mnras/278.1.27

[36] W. Heisenberg, "Introduction to the Unified Field Theory of Elementary Particles,” Max-Planck-Institut für Physik und Astrophysik, Interscience Publishers London, New York, 1966.

[37] V. Dzhunushaliev, Science Echoes, Vol. 4, 2008, pp. 82112. 


\section{Appendix A: Heisenberg's Quantization of Strongly Interacting Fields Applying for Gauge Fields}

In this section we would like to bring some arguments that nonperturbative quantized $\mathrm{SU}(3)$ gauge field tends very quickly to zero. To do so we use the Heisenberg approach [36] for a nonperturbative quantization of a nonlinear spinor field. In quantizing strongly interacting SU(3) gauge fields-via Heisenberg's non-perturbative method [37] one first replaces the classical fields by field operators $A_{\mu}^{B} \rightarrow \hat{A}_{\mu}^{B}$. This yields the following differential equations for the field operators

$$
\partial_{v} \hat{F}^{B \mu v}=0 .
$$

These nonlinear equations for the field operators of the nonlinear quantum fields can be used to determine expectation values for the field operators $\hat{A}_{\mu}^{B}$. Starting with Equation (A1) one can generate an operator differential equation for the product $\hat{A}_{\mu}^{B} \hat{A}^{C}$ consequently allowing the determination of the Green's function $\mathrm{G}_{\mu v}^{B C}$

$$
\begin{aligned}
& \mathrm{G}_{\mu \nu}^{B C}(x, y)=\left\langle Q\left|\hat{A}_{\mu}^{B}(x) \hat{A}_{v}^{C}(y)\right| Q\right\rangle, \\
& \left\langle Q\left|\hat{A}_{\mu}^{B}(x) \partial_{y v} \hat{F}^{C \mu \nu}(x)\right| Q\right\rangle=0
\end{aligned}
$$

where $|Q\rangle$ is a quantum state. However this equation will in it's turn contain other, higher order Green's functions. Repeating these steps leads to an infinite set of equations connecting Green's functions of ever increasing order. Let us note that absolutely similar idea is applied in turbulent hydrodynamics for correlation functions all orders. This construction, leading to an infinite set of coupled, differential equations, does not have an exact, analytical solution and so must be handled using some approximation. The basic approach in this case is to give some physically reasonable scheme for cutting off the infinite set of equations for the Green's functions.

One can use Heisenberg's approach to reduce the initial SU(3) Lagrangian to an effective Lagrangian describing two interacting scalar fields (for details see Ref. [29]). Two scalar fields $\phi$ and $\chi$ appear in such approach. We assume that in the first approximation two points Green's functions are bilinear combinations of scalar fields $\phi$ and $\chi$

$$
\begin{aligned}
\left\langle A_{i}^{a}(x) A_{j}^{b}(y)\right\rangle & \approx c_{m n} \chi^{m}(x) \chi^{n}(y), \\
\left\langle A_{i}^{m}(x) A_{j}^{n}(y)\right\rangle & \approx d_{a b} \phi^{a}(x) \phi^{b}(y), \\
\left\langle A_{0}^{m}(x) A_{0}^{n}(y)\right\rangle & \approx 0
\end{aligned}
$$

where $A_{u}^{a} \in S U(2) \subset S U(3), a=1,2,3$;

$A_{\mu}^{m} \in S U(3) / S U(2), m=4,5,6,7,8 ; c_{m n}, d_{a b}$ are some coefficients.

The next step is the calculation of the 4-points Green's functions. It is assumed that they are a bilinear combination of 2-points Green functions

$$
\begin{aligned}
\left\langle A_{\mu}^{m}(x) A_{\nu}^{n}(y) A_{\alpha}^{p}(z) A_{\beta}^{q}(u)\right\rangle & \\
= & \lambda_{1}\left[\mathrm{G}_{\mu \nu}^{m n} \mathrm{G}_{\alpha \beta}^{p q}+\frac{\mu_{1}^{2}}{4}\left(\delta^{m n} \eta_{\mu \nu} \mathrm{G}_{\alpha \beta}^{p q}+\delta^{p q} \eta_{\alpha \beta} \mathrm{G}_{\mu \nu}^{m n}\right)\right. \\
& \left.+\frac{\mu_{1}^{4}}{16} \delta^{m n} \eta_{\mu \nu} \delta^{p q} \eta_{\alpha \beta}\right]+(\text { permutations of indices) }
\end{aligned}
$$

the same for remaining indexes

$$
\begin{aligned}
\left\langle A_{\mu}^{a}(x) A_{\nu}^{b}(y) A_{\alpha}^{c}(z) A_{\beta}^{d}(u)\right\rangle & \\
= & \lambda_{2}\left[\mathrm{G}_{\mu \nu}^{a b} \mathrm{G}_{\alpha \beta}^{c d}+\frac{\mu_{2}^{2}}{4}\left(\delta^{a b} \eta_{\mu \nu} \mathrm{G}_{\alpha \beta}^{c d}+\delta^{c d} \eta_{\alpha \beta} \mathrm{G}_{\mu \nu}^{a b}\right)\right. \\
& \left.+\frac{\mu_{2}^{4}}{16} \delta^{a b} \eta_{\mu \nu} \delta^{c d} \eta_{\alpha \beta}\right]+(\text { permutations of indices })
\end{aligned}
$$

with some constants $\lambda_{1,2}, \mu_{1,2}$. The assumptions (A3)(A8) allows us to average the SU(3) Lagrangian

$$
\mathrm{L}_{S U(3)}=-\frac{1}{4} F_{\mu \nu}^{A} F^{A \mu \nu},
$$

and bring it to the form

$$
\begin{aligned}
& \mathrm{L}_{\text {eff }}=\left\langle\mathrm{L}_{S U(3)}\right\rangle=\frac{1}{2}\left(\partial_{\mu} \phi^{a}\right)\left(\partial^{\mu} \phi^{a}\right) \\
& +\frac{1}{2}\left(\partial_{\mu} \chi^{m}\right)\left(\partial^{\mu} \chi^{m}\right)-V\left(\phi^{a}, \chi^{m}\right), \\
& V\left(\phi^{a}, \chi^{m}\right) \\
& =\frac{\lambda_{1}}{4}\left(\phi^{a} \phi^{a}-\mu_{1}^{2}\right)^{2}-\frac{\lambda_{2}}{4}\left(\chi^{m} \chi^{m}-\mu_{2}^{2}\right)^{2} \\
& -\frac{1}{2}\left(\phi^{a} \phi^{a}\right)\left(\chi^{m} \chi^{m}\right)
\end{aligned}
$$

with the field equations

$$
\begin{aligned}
& \nabla_{\mu}\left(\nabla^{\mu} \phi^{a}\right)=-\frac{\partial V\left(\phi^{a}, \chi^{m}\right)}{\partial \phi^{a}} \\
& \nabla_{\mu}\left(\nabla^{\mu} \chi^{m}\right)=-\frac{\partial V\left(\phi^{a}, \chi^{m}\right)}{\partial \chi^{m}} .
\end{aligned}
$$

Let us consider the spherically symmetric case $\phi^{a}=k \phi(r), \chi^{a}=k \chi(r)$ where $k$ is some constant. In this case the field equations are

$$
\begin{gathered}
\frac{\mathrm{d}^{2} \phi}{\mathrm{d} r^{2}}+\frac{2}{r} \frac{\mathrm{d} \phi}{\mathrm{d} r}=\phi\left[\chi^{2}+\lambda_{1}\left(\phi^{2}-\mu_{1}^{2}\right)\right], \\
\frac{\mathrm{d}^{2} \chi}{\mathrm{d} r^{2}}+\frac{2}{r} \frac{\mathrm{d} \chi}{\mathrm{d} r}=\chi\left[\phi^{2}+\lambda_{2}\left(\chi^{2}-\mu_{2}^{2}\right)\right] .
\end{gathered}
$$

It is easy to see that asymptoticly the solution has the form 


$$
\begin{aligned}
& \phi(x) \approx m_{1}+\phi_{\infty} \frac{\mathrm{e}^{-\left(r-r_{q}\right) \sqrt{2 \lambda_{1} \mu_{1}^{2}}}}{r}, \\
& \chi(x) \approx \chi_{\infty} \frac{\mathrm{e}^{-\left(r-r_{q}\right) \sqrt{m_{1}^{2}-\lambda_{2} \mu_{2}^{2}}}}{r}
\end{aligned}
$$

where $\phi_{\infty}, \chi_{\infty}, r_{q}$ are constants. We suppose that this solution describes the non-perturbative quantized SU(3) gauge field after the transition from classical phase to quantum one occurs. We see that the non-perturbative quantized gauge field decreases very quickly (exponentially) after transition to the quantum phase and consequently the total mass becomes finite one.

\section{Appendix B: Fitting of Rotational Curve of Gauge Field}

For the fitting of the rotational curve (33) we use the data from the Universal Rotational Curve (27). The fitting equation is equation (33) in the form

$$
\frac{V^{2}}{V_{\text {opt }}^{2}}=A x^{B}+C \text {, }
$$

where

$$
\begin{aligned}
A= & \frac{2}{3} \frac{\alpha^{2}(\alpha-1)(3 \alpha-1)}{2 \alpha-1} \\
& \cdot \frac{c^{2}}{V_{o p t}^{2}} \frac{1}{g^{\prime 2}}\left(\frac{l_{P l}}{r_{0}}\right)^{2}\left(\frac{R_{o p t}}{r_{0}}\right)^{2 \alpha-2} \\
B= & 2 \alpha-2,
\end{aligned}
$$

$$
C=-V_{0}^{2}
$$

and fitted parameters are $A, B, C$. The fitting is carried out using MATHEMATICA package

$$
\begin{aligned}
& A=1.27445, B=0.435011, \\
& C=-0.785905, \frac{L}{L_{*}}=0.5 ; \\
& A=0.656585, B=0.639077, \\
& C=-0.322505, \frac{L}{L_{*}}=1.0 \\
& A=0.428943, B=0.753972, \\
& C=-0.18473, \frac{L}{L_{*}}=1.5
\end{aligned}
$$

after that we can define parameters $\alpha, r_{0}$ and

$$
\begin{aligned}
r_{0} & \approx 1.18 \times 10^{-21} \mathrm{~cm}, V_{0} \approx 78.6 \mathrm{Km} / \mathrm{s}, \\
\alpha & =1.21, \frac{L}{L_{*}}=0.5 ; \\
r_{0} & \approx 2.01 \times 10^{-18} \mathrm{~cm}, V_{0} \approx 32.25 \mathrm{Km} / \mathrm{s}, \\
\alpha & =1.31, \frac{L}{L_{*}}=1.0 ; \\
r_{0} & \approx 8.04 \times 10^{-17} \mathrm{~cm}, V_{0} \approx 18.5 \mathrm{Km} / \mathrm{s}, \\
\alpha & =1.38, \frac{L}{L_{*}}=1.5 .
\end{aligned}
$$

